University of Nebraska - Lincoln

DigitalCommons@University of Nebraska - Lincoln

$1-1-2000$

\title{
A small-system ensemble Monte Carlo simulation of supersaturated vapor: Evaluation of barrier to nucleation
}

K.J. Oh

University of Nebraska-Lincoln

Xiao Cheng Zeng

University of Nebraska-Lincoln, xzeng1@unl.edu

Follow this and additional works at: https://digitalcommons.unl.edu/chemzeng

Part of the Chemistry Commons

Oh, K.J. and Zeng, Xiao Cheng, "A small-system ensemble Monte Carlo simulation of supersaturated vapor: Evaluation of barrier to nucleation" (2000). Xiao Cheng Zeng Publications. 54.

https://digitalcommons.unl.edu/chemzeng/54

This Article is brought to you for free and open access by the Published Research - Department of Chemistry at DigitalCommons@University of Nebraska - Lincoln. It has been accepted for inclusion in Xiao Cheng Zeng Publications by an authorized administrator of DigitalCommons@University of Nebraska - Lincoln. 


\title{
A small-system ensemble Monte Carlo simulation of supersaturated vapor: Evaluation of barrier to nucleation
}

\author{
K. J. Oh and X. C. Zeng ${ }^{\text {a) }}$ \\ Department of Chemistry, University of Nebraska-Lincoln, Lincoln, Nebraska 68588
}

(Received 2 September 1999; accepted 5 October 1999)

\begin{abstract}
A small-system grand canonical ensemble Monte Carlo method is developed to evaluate cluster size distribution and barrier to the nucleation in a supersaturated Lennard-Jones vapor. The theoretical foundation is a physical cluster theory in which the Stillinger cluster is used as a prototypical physical cluster. Using method of Mayer's cluster expansion, the cluster-vapor interaction is effectively taken into account. From a separate canonical ensemble Monte Carlo simulation using a test particle method, the averaged volume of the cluster is obtained and is also incorporated in the small-system ensemble simulation. By this implementation our simulation is computationally more efficient compared to that based on the $n / \mathrm{V}$ Stillinger cluster theory in that instead of searching the saddle point on a two-dimensional free energy surface (a function of cluster size $n$ and volume $\mathrm{v}$ ) one needs only to find the peak on a free energy curve (a function of $n$ only). A comparison with the height of barrier obtained from a large-system ensemble Monte Carlo simulation [K. Oh and X. C. Zeng, J. Chem. Phys. 110, 4471 (1999)] shows that omission of the vapor-cluster attraction can cause an overestimation of the height by several $k_{B} T$. (C) 2000 American Institute of Physics.
\end{abstract}

[S0021-9606(00)50301-8]

\section{INTRODUCTION}

Homogeneous vapor-liquid nucleation is a thermally activated process and the barrier to the nucleation can be characterized by the free energy of formation for the critical cluster. In the classical theory of nucleation ${ }^{1}$ the free energy of formation is calculated based on two assumptions: the center of a cluster behaves like a bulk phase and the surface tension of a cluster is the same as that of an planar surface. Both assumptions become questionable when a cluster contains only a few tens of molecules. In fact, various experiments have shown that the classical theory of nucleation is quite successful in predicting the critical supersaturation of nucleation but not the temperature dependence of the rate of nucleation. To remedy the latter problem, some progress has been made ${ }^{2,3}$ using the nucleation scaling relations. ${ }^{4,5} \mathrm{Re}$ cently, much more attention has been devoted to molecular theory of nucleation and associated method of computer simulation to achieve first-principles prediction of the rate of nucleation for a supersaturated vapor. ${ }^{6-23}$ In all these molecular-based approaches, the determination of clustersize distribution or related free energy of formation has been the central focus.

Methods of computer simulation for study of nucleation can be roughly divided into two families: small-system ensemble simulation for a single cluster ${ }^{8,10-19}$ and conventional large-system ensemble simulation for a supersaturated vapor. ${ }^{20-22}$ For the second family, a large system and number of particles are required to sample the cluster distribution in a supersaturated vapor. This kind of computer simulation mimics real nucleation experiments, where vapor-cluster and cluster-cluster interactions are all taken into account in a realistic fashion. In particular, with molecular dynamics

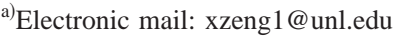

technique one is able to monitor the dynamics of cluster formation and growth in real time. However, these methods can be applied only to study highly supersaturated vapor (typically at a supersaturation above 7). In contrast, methods of small-system ensemble simulation for a single cluster are computationally more efficient. These methods have been applied to compute cluster-size distribution in moderately or even weakly supersaturated vapor (e.g., at a supersaturation of 2). For example, ten Wolde and Frenkel ${ }^{17}$ employed an efficient umbrella-sampling technique in small-system ensemble simulation with which they obtained cluster-size distribution in a Lennard-Jones vapor with a supersaturation less than 2.2. Kusaka et al. ${ }^{14-16}$ developed a small-system grand canonical ensemble method which is particularly efficient for associative fluids such as water. ${ }^{19}$ To evaluate cluster-size distribution they carefully choose the size of a simulation cell such that all molecules in the cell can be viewed as belonging to a single physical cluster. The cell is centered on a marked molecule to avert the problem of overcounting cluster configurations. ${ }^{24}$

Thus far, the interaction between the cluster and vapor has been omitted in most small-system ensemble computer simulations. This omission is reasonable for extremely low vapor density. However, if the vapor density is not so low, the work to create a hole for the cluster in the vapor is not negligible on account of the vapor-cluster interaction. In other cases, such as dipolar fluid (e.g., water) clusters containing an ion, the vapor-cluster interaction can also be quite strong. This vapor-cluster interaction cannot be easily treated by using periodic boundary conditions. ${ }^{16}$

The aim of this work is to describe a small-system ensemble simulation method that effectively incorporates the vapor-cluster interaction in the evaluation of cluster-size distribution and barrier to the nucleation. This method is based on the physical cluster theory of Reiss and 
co-workers ${ }^{6-13}$ where the physical cluster refers to those clusters that contribute significantly to the dynamics of nucleation. To account for the vapor-cluster interaction, Mayer's cluster expansion is applied in the physical cluster theory.

As pointed out by Reiss and co-workers, the physical cluster should be identified in a consistent way to avoid overcounting clusters. In an earlier version of the physical cluster theory, ${ }^{6-10}$ for example, the cluster is described by the $i / \mathrm{v}$ cluster (an extension of Lee-Barker-Abraham cluster ${ }^{25}$ ) which is defined as a group of molecules confined in a rigid spherical cavity with its center coincident with the center-ofmass of the cluster. This $i / \mathrm{V}$ cluster is composed of vapor molecules and a liquid-like core. It turns out that the vapor molecules render the $i / \mathrm{V}$ cluster as not meeting the physical cluster requirements. A later version of physical cluster theory uses a so-called $\alpha$ condition to remove the vapor molecules; the new cluster is termed an $n / \mathrm{v}$ cluster. ${ }^{11}$ The $\alpha$ condition requires that the sum of potential energy of a molecule with other molecules in the cluster is less than $\alpha$ and the sum of potential energy of the molecule with surrounding vapor molecules is greater than $\alpha$. However, Reiss and coworkers recently found ${ }^{12,13}$ that even a large value of $|\alpha|$ cannot prevent molecules within the cluster from decomposing into several distinct fragments (each satisfying the $\alpha$ condition) and moreover, even with the $\alpha$ condition it is still possible to have configurations of a small cluster being part of a larger one. To fix these problems Reiss and co-workers developed the latest version of physical cluster theory-the $n / \mathrm{v}$ Stillinger cluster theory. ${ }^{12,13}$ In this theory, the $\alpha$ condition is replaced by the Stillinger connectivity condition. ${ }^{26}$

In this work, we also designate the Stillinger cluster as an example of the physical cluster. The Stillinger cluster is defined as a group of molecules connected directly and every molecule has at least one nearest neighbor within the distance less than $r_{\mathrm{cl}}$. Note that the Stillinger cluster was also used to identify physical clusters in a large-system ensemble Monte Carlo simulation of nucleation by us. ${ }^{22}$

In Secs. II A and II B we present general expressions for the most probable cluster-size distribution and free energy of formation. These expressions will not be limited only to the Stillinger cluster but can be applied for cluster defined by any physically meaningful criteria. In Sec. III, we examine the two expressions under the assumptions that the vapor is an ideal gas and the cluster has an excluded volume (a model for vapor-cluster interaction). Based on the theoretical formalism in Sec. III, we develop a small-system grand canonical ensemble method in Sec. IV. The cluster free energies of formation are computed via Monte Carlo simulation and shown in Sec. V. Finally, the conclusion is presented in Sec. VI.

\section{THEORETICAL FORMALISM}

\section{A. Most probable cluster-size distribution}

For a supersaturated vapor containing $N$ molecules in volume $V$ at temperature $T$, the total potential energy $U_{N}$ is given by

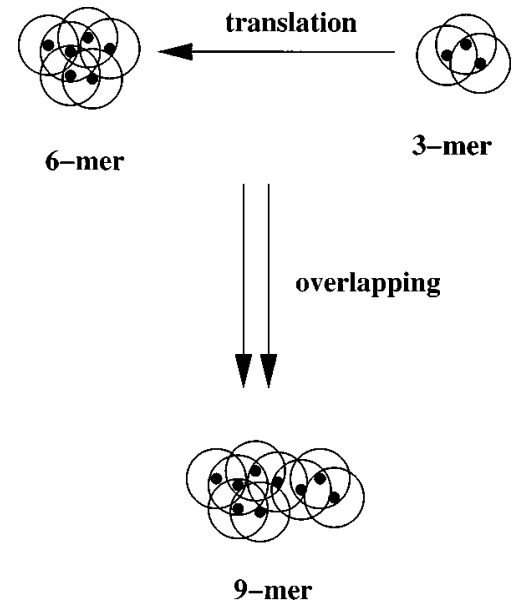

FIG. 1. Schematic illustration of the excluded volume interaction.

$$
U_{N}=\frac{1}{2} \sum_{k}^{N} \sum_{l \neq k}^{N} u_{k l}
$$

where $u_{k l}$ is the pair potential energy between molecule $k$ and $l$; the partition function $Q$ of the system is given by

$$
Q=\int_{V} \frac{\gamma^{N}}{N !} \exp \left(-\beta U_{N}\right) d \mathbf{r}_{1}, \ldots, d \mathbf{r}_{N},
$$

where $\gamma=\left(2 \pi m k_{B} T\right)^{3 / 2} / h^{3}, \beta=1 / k_{B} T$, and $k_{B}$ is the Boltzmann constant. Hereafter, the initial value is 1 for all indexes in the summation sign.

In the physical cluster theory, each configuration of a supersaturated vapor is characterized by a specific clustersize distribution which includes $n_{1}$ monomers, $n_{2}$ dimers,..., $n_{i} i$-mers, $\ldots$, and $n_{i_{\max }} i_{\max }$-mers, where

$$
N=\sum_{i}^{i_{\max }} i n_{i},
$$

and $i_{\max }$ is the upper bound of the cluster size (a constraint) to maintain the supersaturated vapor in metastable equilibrium. Among all possible cluster-size distributions, the most probable one, $\left\{n_{i}\right\}$, is the one that contributes predominantly to $Q$. Therefore, the supersaturated vapor can be envisioned as a mixture of clusters with the most probable size distribution $\left\{n_{i}\right\}$.

An important implication inherent in the physical cluster theory is that the excluded volume interaction among clusters is essential. That is to say, overlapping of two clusters cannot occur while clusters are moving inside the entire volume $V$ because such an overlapping would generate a new cluster as shown in Fig. 1. In any events, there should be no change in cluster numbers for a given $\left\{n_{i}\right\}$.

To obtain $\left\{n_{i}\right\}$ we first rewrite the $Q$ from the viewpoint of physical cluster theory. In doing that, we write $U_{N}$ in terms of intracluster $\left(U_{a}\right)$ and intercluster $\left(U_{a b}\right)$ interactions, i.e.,

$$
U_{N}=\sum_{a}^{M} U_{a}+\frac{1}{2} \sum_{a}^{M} \sum_{b \neq a}^{M} U_{a b}
$$


where $M$ is the total number of clusters in the supersaturated vapor and is given by

$$
M=\sum_{i}^{i_{\max }} n_{i}
$$

We then utilize the Mayer's cluster expansion technique to expand the exponential in $Q$ as

$$
\begin{aligned}
\exp \left(-\beta U_{N}\right)= & \left(\prod_{a}^{M} \exp \left(-\beta U_{a}\right)\right) \\
& \times\left(\prod_{a}^{M} \prod_{b \neq a}^{M} \exp \left(-\frac{\beta U_{a b}}{2}\right)\right) \\
= & \left(\prod_{a}^{M} \exp \left(-\beta U_{a}\right)\right)\left(\prod_{a}^{M} \prod_{b \neq a}^{M}\left(f_{a b}+1\right)\right) \\
= & \left(\prod_{a}^{M} \exp \left(-\beta U_{a}\right)\right) \\
& \times\left(1+\sum_{a}^{M} \sum_{b \neq a}^{M} f_{a b}+\cdots\right)
\end{aligned}
$$

where $f_{a b}$ is the Mayer function, i.e.,

$$
f_{a b}=\exp \left(-\frac{\beta U_{a b}}{2}\right)-1
$$

Assuming physical clusters of the same size are indistinguishable, there will be $N ! / \Pi_{i}^{i_{\text {max }}} n_{i} !(i !)^{n_{i}}$ different ways of permuting the $N$ molecules. Therefore, inserting Eq. (6) into Eq. (2) yields

$$
\begin{aligned}
Q= & \sum_{\left\{n_{i}\right\}}\left(\prod_{i}^{i_{\max }} \frac{q_{0, i}^{n_{i}}}{n_{i} !}\right) \\
& \times\left[1+\sum_{i}^{i_{\max }}\left(n_{i}^{2}-n_{i}\right) F_{i i}+\sum_{i}^{i_{\max }} \sum_{j \neq i}^{i_{\max }} n_{i} n_{j} F_{i j}+\cdots\right],
\end{aligned}
$$

where $q_{0, i}$ is the partition function of an $i$-mer given by

$$
q_{0, i}=\frac{\gamma^{i}}{i !} \int_{V} H\left(\mathbf{r}_{1}, \ldots, \mathbf{r}_{i}\right) \exp \left(-\beta U_{i}\right) d \mathbf{r}_{1}, \ldots, d \mathbf{r}_{i}
$$

In Eq. (9), we add a unit step function $H\left(\mathbf{r}_{1}, \ldots, \mathbf{r}_{i}\right)$ to enforce the criterion of the physical cluster so that the partition of potential energy into intra- and intercluster interaction [as in Eq. (4)] is feasible. If the criterion of the cluster is met, $H=1$ and otherwise, $H=0$. In Eq. (8) one can see that the term in the first parenthesis is simply the partition function of an ideal gas mixture of clusters with size distribution $\left\{n_{i}\right\}$ (i.e., no interaction among all clusters). The term $F_{i j}$ in Eq. (8) is given by

$$
F_{i j}=\frac{\gamma^{i+j}}{i ! j !} \frac{\int_{V} H\left(\mathbf{r}_{1}, \ldots, \mathbf{r}_{i}\right) \exp \left(-\beta U_{i}\right) f_{i j} H\left(\mathbf{r}_{1}, \ldots, \mathbf{r}_{j}\right) \exp \left(-\beta U_{j}\right) d \mathbf{r}_{1}, \ldots, d \mathbf{r}_{i} d \mathbf{r}_{1}, \ldots, d \mathbf{r}_{j}}{q_{0, i} q_{0, j}} .
$$

Thus, $F_{i j}$ is a first-order correction to the ideal gas partition function. Intercluster interactions involving more than two clusters are described by higher order terms in Eq. (8). As such, $Q$ has a form similar to that of the virial expansion for an imperfect gas. In this work, the higher order terms beyond $F_{i j}$ will not be considered.

Although in principle all molecules in the cluster can move within the entire volume $V$ as indicated by the range of integration in Eq. (9), the step function $H\left(\mathbf{r}_{1}, \ldots, \mathbf{r}_{i}\right)$ restricts motion of molecules only within a small region $v$ around the center-of-mass (COM) of the cluster. Let us transform the spatial variables in the laboratory coordinate system to those in the COM coordinate system. Equation (9) can then be written as

$q_{0, i}=\frac{i^{3} \gamma^{i} V}{i !} \int_{\mathrm{V}} H\left(\mathbf{r}_{1}^{\prime}, \ldots, \mathbf{r}_{i-1}^{\prime}\right) \exp \left(-\beta U_{i}\right) d \mathbf{r}_{1}^{\prime}, \ldots, d \mathbf{r}_{i-1}^{\prime}$, where $i^{3}$ comes from the Jacobian of the coordinate transformation, $V$ from the integration over the COM coordinate $\mathbf{R}_{i}$, and therefore the cluster partition function $q_{0, i}^{*}$ in the COM coordinates system is given by

$$
\begin{aligned}
q_{0, i}^{*}= & \frac{i^{(3 / 2)} \gamma^{i-1}}{i !} \int_{\mathrm{V}} H\left(\mathbf{r}_{1}^{\prime}, \ldots, \mathbf{r}_{i-1}^{\prime}\right) \\
& \times \exp \left(-\beta U_{i}\right) d \mathbf{r}_{1}^{\prime}, \ldots, d \mathbf{r}_{i-1}^{\prime}
\end{aligned}
$$

Note that the integration in $q_{0, i}^{*}$ is over a small volume $\mathrm{V}$ as long as $\mathrm{V}$ is larger than the excluded volume of the cluster since $q_{0, i}^{*}$ would not be affected by enlarging the range of volume integration beyond $\mathrm{v}$ due to the reason mentioned above. The same coordinate transformation can be taken for $F_{i j}$ as follows:

$$
F_{i j}=\frac{\gamma^{i+j-2} i^{3 / 2} j^{3 / 2}}{i ! j !} \frac{\int_{\mathrm{v}} H\left(\mathbf{r}_{1}^{\prime}, \ldots, \mathbf{r}_{i-1}^{\prime}\right) \exp \left(-\beta U_{i}\right) f_{i j} H\left(\mathbf{r}_{1}^{\prime}, \ldots, \mathbf{r}_{j-1}^{\prime}\right) \exp \left(-\beta U_{j}\right) d \mathbf{r}_{1}^{\prime}, \ldots, d \mathbf{r}_{i-1}^{\prime} d \mathbf{r}_{1}^{\prime}, \ldots, d \mathbf{r}_{j-1}^{\prime} \int_{V} d \mathbf{R}_{i} d \mathbf{R}_{j}}{V^{2} q_{0, i}^{*} q_{0, j}^{*}}
$$


When $Q$ is known, the Helmholtz free energy $A$ of the supersaturated vapor can be determined as follows:

$$
\begin{aligned}
A= & -k_{B} T \ln Q \\
= & \sum_{i}\left(k_{B} T \ln \frac{n_{i} !}{q_{0, i}}\right) \\
& -k_{B} T \ln \left[1+\sum_{i}^{i_{\max }}\left(n_{i}^{2}-n_{i}\right) F_{i i}+\sum_{i}^{i_{\max }} \sum_{j \neq i}^{i_{\max }} n_{i} n_{j} F_{i j}\right] \\
= & \sum_{i} n_{i} k_{B} T\left(\ln \frac{n_{i}}{q_{0, i}}-1\right)-k_{B} T \sum_{i}^{i_{\max }}\left(n_{i}^{2}-n_{i}\right) F_{i i} \\
& -k_{B} T \sum_{i}^{i_{\max }} \sum_{j \neq i}^{i_{\max }} n_{i} n_{j} F_{i j} .
\end{aligned}
$$

In deriving the last equation in (14) we used the Stirling's formula for $n_{i}$ !; we also expanded the logarithm and truncated the expansion at the second-order term. Minimizing $A$ with respect to the number of cluster $n_{i}$ under the constraint of Eq. (3) results in the well-known law of mass action

$$
\alpha_{i}=i \alpha_{1},
$$

for all $i$ where

$$
\alpha_{i}=k_{B} T \ln \frac{n_{i}}{q_{0, i}}-k_{B} T\left(2 n_{i}-1\right) F_{i i}-k_{B} T \sum_{j \neq i}^{i_{\max }} n_{j} F_{i j} .
$$

From the standard definition of chemical potential

$$
\mu_{i}\left(p_{i}\right)=\left(\frac{\partial A}{\partial n_{i}}\right)_{T, V, n_{j}}, \quad(j \neq i),
$$

one can easily verify that $\alpha_{i}$ is just $\mu_{i}\left(p_{i}\right)$, the chemical potential of an $i$-mer at its partial pressure $p_{i}$.

\section{B. Cluster free energy of formation}

We define the free energy of formation $\Delta G_{i}$ for an $i$-mer ${ }^{10}$ as the change in Gibbs free energy for the following reaction at the partial pressure of monomer $p_{1}$ :

$$
i A_{1} \rightleftharpoons A_{i},
$$

where $A_{1}$ and $A_{i}$ symbolize the monomer and $i$-mer, respectively. In other words, $\Delta G_{i}$ is the reversible work required to transform $i$ monomers to one $i$-mer at $p_{1}$

$$
\Delta G_{i}=\mu_{i}\left(p_{1}\right)-i \mu_{1}\left(p_{1}\right) .
$$

Note that $\Delta G_{i}$ can also be defined as the chemical potential difference at partial pressure $p_{i}$ and $p_{1}$, i.e.,

$$
\Delta G_{i}=\mu_{i}\left(p_{1}\right)-\mu_{i}\left(p_{i}\right) .
$$

The term $\mu_{i}\left(p_{1}\right)$ in both Eqs. (19) and (20) can be expressed (approximately) using a formula for ideal gas

$$
\mu_{i}\left(p_{1}\right) \approx \mu_{i}\left(p_{i}\right)+k_{B} T \ln \frac{n_{1}}{n_{i}},
$$

which can be derived from integration of the Gibss-Duhem equation. Substituting Eq. (21) in Eqs. (19) and (20) yields, respectively,

$$
\Delta G_{i}=\mu_{i}\left(p_{i}\right)+k_{B} T \ln \frac{n_{1}}{n_{i}}-i \mu_{1}\left(p_{1}\right),
$$

and

$$
\Delta G_{i}=k_{B} T \ln \frac{n_{1}}{n_{i}} .
$$

In the classical theory of nucleation, the Gibbs free energy (or grand canonical free energy $\Omega$ ) of droplet formation is defined as

$$
\begin{aligned}
\Delta G & =\Delta \Omega, \\
& =-p_{l} V_{l}+p_{g} V_{l}+\sigma A, \\
& =N_{l}\left(\mu_{l}\left(p_{g}\right)-\mu_{g}\left(p_{g}\right)\right)+\sigma A,
\end{aligned}
$$

where $N_{l}$ is the number of molecules in the droplet (or cluster), $\mu_{l}\left(p_{l}\right)$, the chemical potential of droplet, $V_{l}$, the droplet volume, and $p_{l}$, the pressure inside the droplet; $\mu_{g}\left(p_{g}\right)$ is the chemical potential of the supersaturated vapor at pressure $p_{g}$, which equals $\mu_{l}\left(p_{l}\right)$ because of the (unstable) equilibrium condition; $A$ is the surface area of the droplet, and $\sigma$ is the surface tension. Equation (26) is readily compared with the rigorous ones, Eqs. (19) or (20), in which $\mu_{i}\left(p_{1}\right)$ incorporates the surface free energy implicitly.

\section{AN INTERCLUSTER POTENTIAL MODEL}

In Sec. II, we presented a general formalism for the most probable cluster-size distribution and the Stillinger cluster free energy of formation. In this section, we apply this formalism to a supersaturated vapor. For simplicity, we first make an approximation that $F_{11}=0$ and $F_{i j}=0$ for all $i$ and $j$ except $i \neq 1$ or $j \neq 1$, i.e., only $F_{1 i}=F_{i 1} \neq 0$ for $i$ $=2, \ldots, i_{\max }$. Physically, this approximation implies that clusters interact only with vapor molecules (monomers) and the vapor itself is an ideal gas (no monomer-monomer interaction). The approximation is a reasonable one if the vapor density is relatively low (e.g., not close to the critical point). Next, we propose a simple intercluster potential model

$$
\begin{aligned}
U_{i j} & =\infty \quad \text { if two clusters overlap } \\
& =i U_{1} \quad \text { otherwise, }
\end{aligned}
$$

where $U_{1}$ is a constant, which represents an effective attractive interaction between vapor and cluster per cluster molecule. This simple model entails an excluded volume interaction (see Fig. 1) as well as a mean-field-like effective van der Waals attraction between vapor and cluster. In other word, clusters possess a hard core and yet the shape of clusters constantly fluctuates. Thus, from Eqs. (13) and (27) we obtain 
$F_{i 1}=\frac{i^{3 / 2} \gamma^{i-1}}{i !} \frac{\int_{\mathrm{v}} H\left(\mathbf{r}_{1}^{\prime}, \ldots, \mathbf{r}_{i}^{\prime}\right) \exp \left(-\beta U_{i}\right) d \mathbf{r}_{1}^{\prime}, \ldots, d \mathbf{r}_{i-1}^{\prime} \int_{V} f_{i 1} d \mathbf{R}_{1} d \mathbf{R}_{i}}{V^{2} q_{0, i}^{*} q_{0,1}^{*}}$,

where the integration over the COM coordinates yields

$$
\begin{aligned}
\int_{V} f_{i 1} d \mathbf{R}_{1} d \mathbf{R}_{i} & =\int_{V} f_{i 1} d \mathbf{R}_{1} d \mathbf{R}_{i 1} \\
& =V\left(\int_{\text {overlap }} f_{i 1} d \mathbf{R}_{i 1}+\int_{\text {nonoverlap }} f_{i 1} d \mathbf{R}_{i 1}\right) \\
& =V\left[-\tau_{i}-\frac{\beta i U_{1}}{2}\left(V-\tau_{i}\right)\right] .
\end{aligned}
$$

In Eq. (29), $\mathbf{R}_{i 1}=\mathbf{R}_{1}-\mathbf{R}_{i}, \tau_{i}$ is the excluded volume of an $i$-mer, and $f_{i 1} \approx-\beta i U_{1} / 2$ outside the excluded volume $\tau_{i}$, that is, in the nonoverlap region. For the Stillinger cluster, $\tau_{i}$ is the volume occupied by all molecules of the cluster where each molecule has an excluded volume of a sphere with diameter $2 r_{\mathrm{cl}}$ (See Fig. 1 for a schematic view in two dimensions). Combining Eq. (29) with (28) leads to a simple expression

$F_{i 1}=-\frac{\left\langle\tau_{i}\right\rangle}{V}\left(1-\frac{\beta i U_{1}}{2}\right)-\frac{\beta i U_{1}}{2} \approx-\frac{\left\langle\tau_{i}\right\rangle}{V}-\frac{\beta i U_{1}}{2}$,

where

$\left\langle\tau_{i}\right\rangle$

$$
=\frac{i^{3 / 2} \gamma^{i-1}}{i !} \frac{\int_{\mathrm{v}} \tau_{i} H\left(\mathbf{r}_{1}^{\prime}, \ldots, \mathbf{r}_{i-1}^{\prime}\right) \exp \left(-\beta U_{i}\right) d \mathbf{r}_{1}^{\prime}, \ldots, d \mathbf{r}_{i-1}^{\prime}}{q_{0, i}^{*}} .
$$

Here, $\left\langle\tau_{i}\right\rangle$ denotes a canonical average of $\tau_{i}$ over all cluster configurations, which means that the excluded volume can be viewed as a statistical mechanics average quantity of the cluster.

Using Eqs. (30) and (31) one can calculate $\mu_{i}\left(p_{i}\right)$ via Eqs. (16) or (17), that is

$$
\begin{aligned}
\mu_{i}\left(p_{i}\right) & =k_{B} T \ln \frac{n_{i}}{q_{0, i}}+k_{B} T n_{1}\left(\frac{\left\langle\tau_{i}\right\rangle}{V}+\frac{\beta i U_{1}}{2}\right) \\
& =k_{B} T \ln \frac{n_{i}}{q_{0, i}}+p_{1}\left\langle\tau_{i}\right\rangle+\frac{n_{1} i U_{1}}{2} \\
& =-k_{B} T \ln i^{3 / 2} \gamma V+k_{B} T \ln \frac{n_{i}}{q_{0, i}^{*}}+p_{1}\left\langle\tau_{i}\right\rangle+i U_{0},
\end{aligned}
$$

where the first term in Eq. (32) represents the translational free energy of the $i$-mer in $V$, the second term represents the excess chemical potential of the $i$-mer of an ideal mixture at $p_{1}$, the third term represents the work to create the excluded volume due to the vapor-cluster interaction, and the fourth term is due to the attractive interactions between the cluster and the vapor; $U_{0}=n_{1} U_{1} / 2$ represents a mean-field-like averaged interaction energy of the cluster with the $n_{1}$ monomers in vapor. Similarly, one can obtain

$$
\begin{aligned}
\mu_{1}\left(p_{1}\right) & =k_{B} T \ln \frac{n_{1}}{q_{0,1}}+k_{B} T \sum_{i \neq 1} n_{i}\left(\frac{\left\langle\tau_{i}\right\rangle}{V}+\frac{\beta i U_{1}}{2}\right) \\
& \approx k_{B} T \ln \frac{n_{1}}{q_{0,1}} \\
& =k_{B} T \ln \frac{n_{1}}{\gamma V} \\
& =k_{B} T \ln \frac{p_{1}}{\gamma k_{B} T},
\end{aligned}
$$

where we assume $\mu_{1}$ is essentially the chemical potential of an ideal monomer gas.

Substituting Eqs. (32) and (33) into (22) we obtain the cluster free energy of formation

$$
\begin{aligned}
\Delta G_{i}= & -k_{B} T \ln i^{3 / 2} \gamma V+k_{B} T \ln \frac{n_{1}}{q_{0, i}^{*}}+p_{1}\left\langle\tau_{i}\right\rangle+i U_{0} \\
& -i k_{B} T \ln \frac{p_{1}}{\gamma k_{B} T} .
\end{aligned}
$$

Note that $\Delta G_{i}$ as in Eq. (34) was also derived by Reiss and co-workers in $i / \mathrm{v}$ cluster, ${ }^{6-10} n / \mathrm{v}$ cluster, ${ }^{11}$ and $n / \mathrm{v}$ Stillinger cluster theory. ${ }^{12,13}$ The crucial difference is that we separately determine the cluster volume $\left\langle\tau_{i}\right\rangle$ from a canonical average of the instantaneous excluded volume. Therefore, in our approach we are free from searching for a saddle point on the free energy surface spanned by the number of molecules of the cluster $n$ and the volume of cluster $\mathrm{V}$.

\section{SMALL-SYSTEM GRAND CANONICAL ENSEMBLE}

Based on the physical cluster theory and the model of vapor-cluster interaction described in the previous section, we obtain the most probable number of $i$-mers

$$
n_{i}=i^{3 / 2} \gamma V q_{0, i}^{*} e^{\beta i \mu_{1}} e^{-\beta p_{1}\left\langle\tau_{i}\right\rangle} e^{-\beta i U_{0}},
$$

from Eq. (32) using the law of mass action $\left(\mu_{i}=i \mu_{1}\right)$. By summing $n_{i}$ for all $i$, we also obtain the total number of clusters in $V$,

$$
M=\sum_{i=1}^{i_{\max }} i^{3 / 2} \gamma V q_{0, i}^{*} e^{\beta i \mu_{1}} e^{-\beta p_{1}\left\langle\tau_{i}\right\rangle} e^{-\beta i U_{0}} .
$$

Thus, we arrive at two key results of this work, the probability of finding $i$-mer in $V$, i.e., the cluster-size distribution

$$
\begin{aligned}
P_{i} & \equiv \frac{n_{i}}{M} \\
& =\frac{i^{3 / 2} \gamma V q_{0, i}^{*} e^{\beta i \mu_{1}} e^{-\beta p_{1}\left\langle\tau_{i}\right\rangle} e^{-\beta i U_{0}}}{M},
\end{aligned}
$$

and the cluster free energy of formation from Eq. (23) 


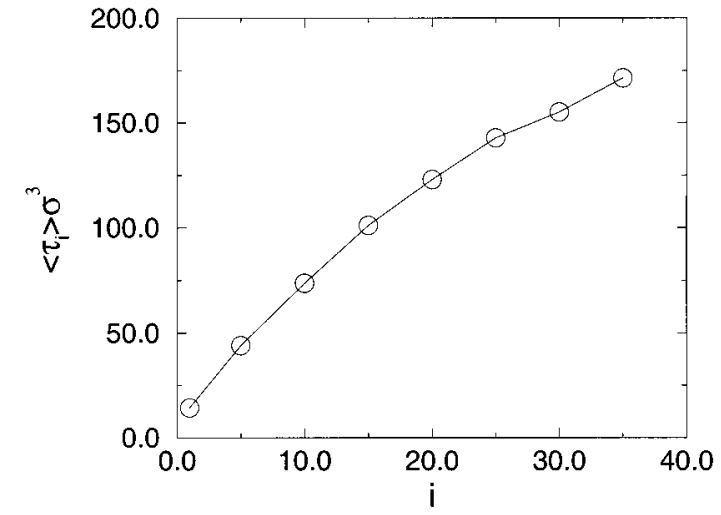

FIG. 2. $\left\langle\tau_{i}\right\rangle$ as a function of $i$ at $k_{B} T / \epsilon=0.67$.

$$
\Delta G_{i}=-k_{B} T \ln \frac{P_{i}}{P_{1}} .
$$

In Eq. (37), $M$ can be viewed as a partition function of a grand canonical ensemble. By taking a Monte Carlo simulation in that ensemble, a molecule can be added to or removed from the cluster while fixing the COM of the cluster. Therefore, from the simulation one can directly generate $P_{i}$ and then calculate $\Delta G_{i}$ from Eq. (38).

The term $p_{1}\left\langle\tau_{i}\right\rangle$ in Eq. (37) is due to the excluded volume interaction with the vapor molecules (monomers) and $i U_{0}$ is due to the attractive interactions between the cluster and the vapor. In the small-system grand canonical ensemble Monte Carlo approach by Kusaka et al., ${ }^{14,15}$ the vaporcluster interaction was neglected. For water (the system Kusaka et al. studied), its contribution to $P_{i}$ is indeed negligible because the vapor density is so low that the term $p_{1}\left\langle\tau_{i}\right\rangle$ and $i U_{0}$ is nearly equal to zero.

Note that in our approach it is not required to map the small-system ensemble simulation results onto macroscopic volume $V^{27-30}$ to obtain the free energy of formation because $P_{i}$ is the probability in $V$, not in $\mathrm{V}$. In fact, the free energy of formation does not depend on the $v$ at all.

\section{MONTE CARLO SIMULATION}

We performed both canonical and grand canonical Monte Carlo (MC) simulations at the reduced temperature $k_{B} T / \epsilon=0.67$ for a Lennard-Jones system with the parameter $\epsilon=119.8 \mathrm{~K}$ and $\sigma=3.405 \AA$ of argon.

First, in the canonical ensemble MC simulation a molecule is selected sequentially and displaced randomly. This displacement is equally divided by $i-1$; each of all the other $i-1$ molecules is then displaced by this amount in the opposite direction to fix the COM of the cluster. Thus, another necessary scheme in addition to the conventional Metropolis rejection scheme is that a trial move would be rejected if the Stillinger cluster criterion is not met. Here, we used the usual value of $r_{\mathrm{cl}}=1.5 \sigma^{13,17,22}$ as the criterion of connectivity in the Stillinger cluster. In order to calculate the excluded volume of the cluster, at every MC step, a ghost molecule (or test particle $)$ is inserted randomly within a volume of [ $i$ $\left.+1) r_{\mathrm{cl}}\right]^{3}$ and then checked if it is connected to the cluster. By calculating the average probability $\xi$ through many inser-

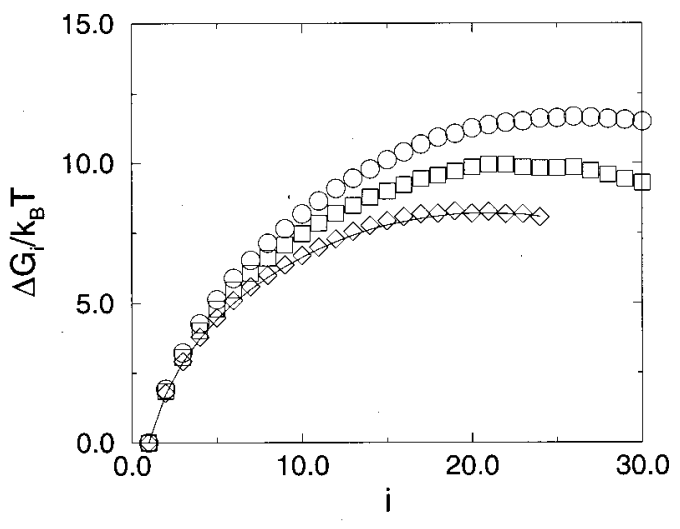

FIG. 3. $\Delta G_{i}$ as a function of $i$ at $k_{B} T / \epsilon=0.67$ and $p_{1} \sigma^{3} / k_{B} T=0.01166$ for $U_{0} / \epsilon=0$ (circle), -0.05 (square), -0.1 (diamond). Solid line is from a large-system ensemble Monte Carlo simulation at $N \sigma^{3} / V=0.023$ using $i_{\max }=30$ and $4.5 \sigma$ as the potential cutoff distance.

tions, we estimated the volume via equation $\tau_{i}=\xi \mathrm{v}$. The insertion will be stopped if the relative error in the calculated volume is less than $10^{-3}$. Figure 2 shows $\left\langle\tau_{i}\right\rangle$ as a function of $i$ averaged over 50000 MC steps after 50000 MC steps for equilibration. We calculated $\left\langle\tau_{i}\right\rangle$ only for $i=1,5,10,15$, 20, 25, 30, and 35; for other $i$, we used 3-point polynomial interpolation to estimate $\left\langle\tau_{i}\right\rangle$.

Next, in the grand canonical simulation we also applied the COM constraint upon adding and removing a molecule from the cluster in addition to MC moves as in the canonical ensemble simulation. Suppose a molecule is added at an arbitrary position $\mathbf{r}_{\text {add }}$ within a volume (the size of this volume will be discussed in the next paragraph). Then, all the preexisting $i$ molecules are displaced by $-\mathbf{r}_{\text {add }} / i$ in order to fix the COM. If the resulting $i+1$ molecules satisfy the cluster criterion, the addition is then accepted. Similarly, if a molecule at position $\mathbf{r}_{\text {rem }}$ is removed, the remaining $i-1$ molecules are displaced by $\mathbf{r}_{\text {rem }} /(i-1)$ and the cluster criterion is checked for these $i-1$ molecules. If the cluster criterion is satisfied, the removal of the molecule is accepted.

Practically, it is computationally inefficient to calculate $P_{i}$ for all $i$ in one simulation because the statistics at the critical cluster size would be very poor. Instead, one can divide the size range of cluster, e.g., from 1 to $i_{\max }$, into several intervals and perform one simulation for each interval. The probability distribution from each interval is then patched together by requiring the total distribution function to be continuous. ${ }^{14}$ In this calculation, the size range is divided into $[1,3],[3,6],[6,9],[9,12],[12,15],[15,18],[18$, 21], [21, 24], [24, 27], [27, 30]. The simulation at each interval is stopped if the number of occurrence of the least frequent cluster is at least $10^{4}$ after the equilibration for $10^{5}$ MC cycles (each MC cycle is composed of $i$ molecule displacement and one addition or removal). At each interval, a volume $\left[\left(n_{f}+1\right) r_{\mathrm{cl}}\right]^{3}$ is used for molecule addition where $n_{f}$ is the largest size of cluster within that interval. This volume is chosen to ensure that outside the volume the cluster criterion cannot be satisfied.

Once $P_{i}$ is obtained, the cluster free energies of formation $\Delta G_{i}$ can be determined using Eq. (38). Figure 3 shows 
the calculated $\Delta G_{i}$ as a function of $i$ for $U_{0} / \epsilon=0,-0.05$, and -0.1 at the scaled pressure $p_{1} \sigma^{3} / k_{B} T=0.01166$. This pressure is close to that estimated from the monomer number in the previous large-system ensemble Monte Carlo simulation $^{22}$ for a supersaturated vapor at a scaled density of $N \sigma^{3} / V=0.023$ with using $i_{\max }=30$ and $4.5 \sigma$ as the potential cutoff distance. When $U_{0}=0, \Delta G$ of the critical cluster, i.e., the barrier to the nucleation, is about $3 k_{B} T$ higher compared to the previous simulation ${ }^{22}$ (solid line in Fig. 3). However, the $\Delta G_{i}$ curve nearly overlaps the solid line as $U_{0} / \epsilon$ is set to be -0.1 , indicating the van der Waals attraction between vapor and cluster can contribute an order of a few $k_{B} T$ to the barrier to the nucleation. ${ }^{31}$

\section{CONCLUSION}

From the view that the supersaturated vapor is essentially a mixture of clusters of various sizes, we reformulate the physical cluster theory using Mayer's cluster expansion technique to take into account the vapor-cluster interaction. The expression for cluster free energy of formation (defined here as the reversible work to transform $i$ monomers into an $i$-mer while keeping the monomer pressure at $p_{1}$ ) is obtained. In this expression, we also incorporate the cluster volume, calculated from a canonical average of the instantaneous excluded volume of clusters. This frees us from searching for the saddle point on the free energy surface spanned by the number of molecules in the cluster and its volume, which is computationally more demanding. ${ }^{12,13}$

Based on the physical cluster theory, we devise a grand canonical ensemble Monte Carlo simulation method to evaluate the cluster-size distribution and the free energy of formation. In our simulations, because the translational move of the cluster over the macrovolume is effectively included, we do not need to map the simulation results to the macrovolume. Several previous small-system grand canonical ensemble MC simulations required this mapping. ${ }^{27-30}$ Finally, we are encouraged that the obtained barrier to the nucleation is very close to the one obtained from a large-system ensemble Monte Carlo simulation for a supersaturated vapor at the same supersaturation and temperature. ${ }^{22}$

\section{ACKNOWLEDGMENTS}

X.C.Z. thanks the National Science Foundation for support of this work.

${ }^{1}$ F. F. Abraham, Homogeneous Nucleation Theory (Academic, New York, 1974).

${ }^{2}$ V. Talanquer, J. Chem. Phys. 106, 9957 (1997).

${ }^{3}$ R. McGraw and A. Laaksonen, J. Chem. Phys. 106, 5284 (1997).

${ }^{4}$ R. McGraw and A. Laaksonen, Phys. Rev. Lett. 76, 2754 (1996).

${ }^{5}$ K. Koga and X. C. Zeng, J. Chem. Phys. 110, 3466 (1999).

${ }^{6}$ H. M. Ellerby, C. L. Weakliem, and H. Reiss, J. Chem. Phys. 95, 9209 (1991).

${ }^{7}$ H. M. Ellerby and H. Reiss, J. Chem. Phys. 97, 5766 (1992).

${ }^{8}$ C. L. Weakliem and H. Reiss, J. Chem. Phys. 99, 5374 (1993).

${ }^{9}$ C. L. Weakliem and H. Reiss, J. Chem. Phys. 101, 2398 (1994).

${ }^{10}$ K. J. Oh, X. C. Zeng, and H. Reiss, J. Chem. Phys. 107, 1242 (1997).

${ }^{11}$ P. Schaaf, B. Senger, and H. Reiss, J. Phys. Chem. 101, 8740 (1997).

${ }^{12}$ See, for example, B. Senger, P. Schaaf, D. S. Corti, R. Bowles, J.-C. Voegel, and H. Reiss, J. Chem. Phys. 110, 6421 (1999), and references therein.

${ }^{13}$ B. Senger, P. Schaaf, D. S. Corti, R. Bowles, D. Pointu, J.-C. Voegel, and H. Reiss, J. Chem. Phys. 110, 6438 (1999).

${ }^{14}$ I. Kusaka, Z.-G. Wang, and J. H. Seinfeld, J. Chem. Phys. 108, 3416 (1998).

${ }^{15}$ I. Kusaka and D. W. Oxtoby, J. Chem. Phys. 110, 5249 (1999).

${ }^{16}$ I. Kusaka, D. W. Oxtoby, and Z.-G. Wang, J. Chem. Phys. (submitted).

${ }^{17}$ P. R. ten Wolde and D. Frenkel, J. Chem. Phys. 109, 9901 (1998).

${ }^{18}$ P. R. ten Wolde, D. W. Oxtoby, and D. Frenkel, J. Chem. Phys. 111, 4762 (1999).

${ }^{19}$ G. T. Gao, K. J. Oh, and X. C. Zeng, J. Chem. Phys. 110, 2533 (1999).

${ }^{20}$ K. Yasuoka and M. Matsumoto, J. Chem. Phys. 109, 8451 (1998).

${ }^{21}$ K. Yasuoka and M. Matsumoto, J. Chem. Phys. 109, 8463 (1998).

${ }^{22}$ K. J. Oh and X. C. Zeng, J. Chem. Phys. 110, 4471 (1999).

${ }^{23}$ G. K. Schenter, S. M. Kathmann, and B. C. Garett, Phys. Rev. Lett. 82, 3484 (1999).

${ }^{24}$ Reiss and co-workers (Refs. 27-30) pointed out that the center of the simulation cell should be fixed on the center-of-mass of the cluster. Otherwise, the cluster-size distribution will include an extra factor resulting from the center-of-mass fluctuation (Ref. 32).

${ }^{25}$ J. K. Lee, J. A. Barker, and F. F. Abraham, J. Chem. Phys. 58, 3166 (1973).

${ }^{26}$ F. H. Stillinger, J. Chem. Phys. 38, 1486 (1963).

${ }^{27}$ H. Reiss, J. Mol. Struct. 485-486, 465 (1999).

${ }^{28}$ H. Reiss and R. K. Bowles, J. Chem. Phys. (submitted).

${ }^{29}$ H. Reiss and R. K. Bowles, J. Chem. Phys. (submitted).

${ }^{30}$ R. K. Bowles, J. Chem. Phys. (submitted).

${ }^{31} \mathrm{~A}$ mean-field estimation of vapor-cluster (size $\left.i\right)$ interaction $i U_{0}$ can be made via the equation $8 \pi^{2} \rho_{g} \rho_{l} \iiint u_{\mathrm{LJ}}\left(\left|\mathbf{r}-\mathbf{r}^{\prime}\right|\right) d \cos (\theta) r^{\prime 2} d r^{\prime} r^{2} d r$, where $u_{\mathrm{LJ}}$ is the Lennard-Jones potential and $\theta$ is the polar angle.

${ }^{32}$ K. J. Oh and X. C. Zeng, J. Chem. Phys. 108, 4683 (1998). 